\title{
MEDICATIONS REGISTERED IN WESTERN EUROPE FOR VARROATOSIS CONTROL
}

\author{
W. RITTER \\ Thierhygienisches Institut Am Moosweiher 2 D-7800 Freiburg
}

Since varroatosis first appeared in Europe a great deal of research was made for substances both effective against Varroa mites but well tolerated by bees. Of the large number found, only a few are actually used in practical beekeeping. In almost all countries of Western Europe bee medicaments must meet the criteria of general veterinary medicaments. Possible toxic and mutagenic effects upon humans are of particular importance for the process of registration. Besides the toxicological studies, tests concerning residues in bee products and dangers to the user are performed.

The medicaments listed in the table were tested in the country of registration for their effectivity, bee tolerance and harmlessness for user and consumer.

\section{ÜBERSICHT DER IN WESTEUROPA ZUR BEKÄMPFUNG DER VARROATOSE ZUGELASSENEN MEDIKAMENTE}

Mit dem ersten Auftreten der Varroatose wurden auch in West-Europa verstärkt Mittel gesucht, die ausreichend wirksam gegen die Varroamilben und gleichzeitig gut bienenverträglich sind. Aus der großen Zahl dieser Substanzen sind nur wenige bis zur Anwendung beim Imker gelangt. In fast allen Ländern West-Europas müssen Bienenarzneimittel als Tierarzneimittel zugelassen werden. Bei der Zulassung wird besonders auf mögliche toxische und mutagene Wirkungen im Humanbereich geachtet. Neben den hierfür notwendigen Studien müssen Unterlagen über mögliche Rückstände in Bienenprodukten und Gefahren für den Anwender eingereicht werden. Die in der Tabelle aufgeführten Medikamente wurden in den jeweiligen Ländern, in denen eine Zulassung erfolgte, bei vorgeschriebener Anwendung als ausreichend wirksam, gut bienenverträglich und unbedenklich für Verbraucher und Anwender beurteilt.

\section{PANORAMA DES MÉDICAMENTS AUTORISÉS EN EUROPE OCCIDENTALE POUR TRAITER LA VARROATOSE}

Lorsque la varroatose a fait son apparition en Europe on a cherché des substances qui soient à la fois efficaces contre l'acarien Varroa et bien supportées par les abeilles. 
TABL. 1. - Medicinal treatments $\mathrm{A}=$ Austria, $\mathrm{B}=$ Belgium, $\mathrm{Ch}=$ Switzerland, $\mathrm{D}=$ Federal Republic of Germany

\begin{tabular}{|c|c|c|c|c|}
\hline Commercial name & FOLBEX-VA & $\begin{array}{l}\text { Formic acid plates } \\
\text { (IMP) }\end{array}$ & Perizin & $\begin{array}{l}\text { Antivarroa-Sche- } \\
\text { ring }\end{array}$ \\
\hline Manufacturer & Ciba Geigy & Klinger & Bayer & Schering \\
\hline Active ingredient & Bromopropylate & Formic acid & Cumaphos & Amitraz \\
\hline Form of application & Fumigant strips & evaporating plates & $\begin{array}{l}\text { systemic functioning } \\
\text { liquid }\end{array}$ & aerosol \\
\hline Mode of application & Fumigation & evaporation & trickled on & aerosol steam \\
\hline $\begin{array}{l}\text { Dosage per treat- } \\
\text { ment and colony }\end{array}$ & 1 strip & $\begin{array}{l}1 \text { plate per super } \\
\text { ( } 20 \mathrm{~g} \text { formic acid) }\end{array}$ & $\begin{array}{l}1 \mathrm{ml} \text { liquid in } 49 \mathrm{ml} \\
\text { water }\end{array}$ & $\begin{array}{l}20 \mathrm{ml} \text { of liquid in } 1 \mathrm{l} \\
\text { water }\end{array}$ \\
\hline $\begin{array}{l}\text { Frequency or period } \\
\text { of use }\end{array}$ & 4 times & 4 times for 12 hours & 2 times & $\begin{array}{l}2 \text { times for } 1 \text { to } 2 \\
\text { minutes }\end{array}$ \\
\hline $\begin{array}{l}\text { Interval between ap- } \\
\text { plications }\end{array}$ & 4 days & 7 days & 7 days & 7 days \\
\hline $\begin{array}{l}\text { External temperatu- } \\
\text { re limits }\end{array}$ & $\min 8{ }^{\circ} \mathrm{C}$ & $\begin{array}{l}\min 12{ }^{\circ} \mathrm{C} \\
\max 25^{\circ} \mathrm{C}\end{array}$ & $\min 5^{\circ} \mathrm{C}$ & $\min 8^{\circ} \mathrm{C}$ \\
\hline $\begin{array}{l}\text { Time of day for treat- } \\
\text { ment }\end{array}$ & evening & $\begin{array}{l}\text { all day preferably } \\
\text { evening }\end{array}$ & all day & morning or evening \\
\hline Special preparations & $\begin{array}{l}\text { insert strips through } \\
\text { hive entrance or in } \\
\text { empty frame close } \\
\text { flight entrance for } \\
1 / 2 \text { hour }\end{array}$ & $\begin{array}{l}\text { plates are placed } \\
\text { above frames or on } \\
\text { bottom board }\end{array}$ & $\begin{array}{l}\text { solution is trickled } \\
\text { on bees between } \\
\text { combs }\end{array}$ & $\begin{array}{l}\text { introduce aerosols } \\
\text { through hive en- } \\
\text { trance or from top, } \\
\text { gasmask should be } \\
\text { worn during appli- } \\
\text { cation }\end{array}$ \\
\hline Registered & $\begin{array}{l}\mathrm{B}, \mathrm{D}, \mathrm{E}, \mathrm{F}, \mathrm{GR}, \mathrm{I}, \\
\mathrm{L}, \mathrm{NL}, \mathrm{CH}, \mathrm{A}\end{array}$ & D & $\underset{\text { A }}{\text { B, D, E, F, L, NL, }}$ & $\mathrm{F}$ \\
\hline $\begin{array}{l}\text { Registration submit- } \\
\text { ted }\end{array}$ & & $\begin{array}{l}\text { registration not ne- } \\
\text { cessary in several } \\
\text { countries }\end{array}$ & GR, I & \\
\hline Literature & $\begin{array}{l}\text { RitTER and Per- } \\
\text { SCHIL (1983) }\end{array}$ & $\begin{array}{l}\text { WACHENDÖRFER et } \\
\text { al. }(1985)\end{array}$ & RITTER (1986) & Colin et al. (1983) \\
\hline
\end{tabular}


ainst Varroa jacobsoni Oud.

$=$ Spain, $\mathrm{F}=$ France, $\mathrm{GR}=$ Greece, $\mathrm{L}=$ Luxemburg, $\mathrm{NL}=$ Netherlands

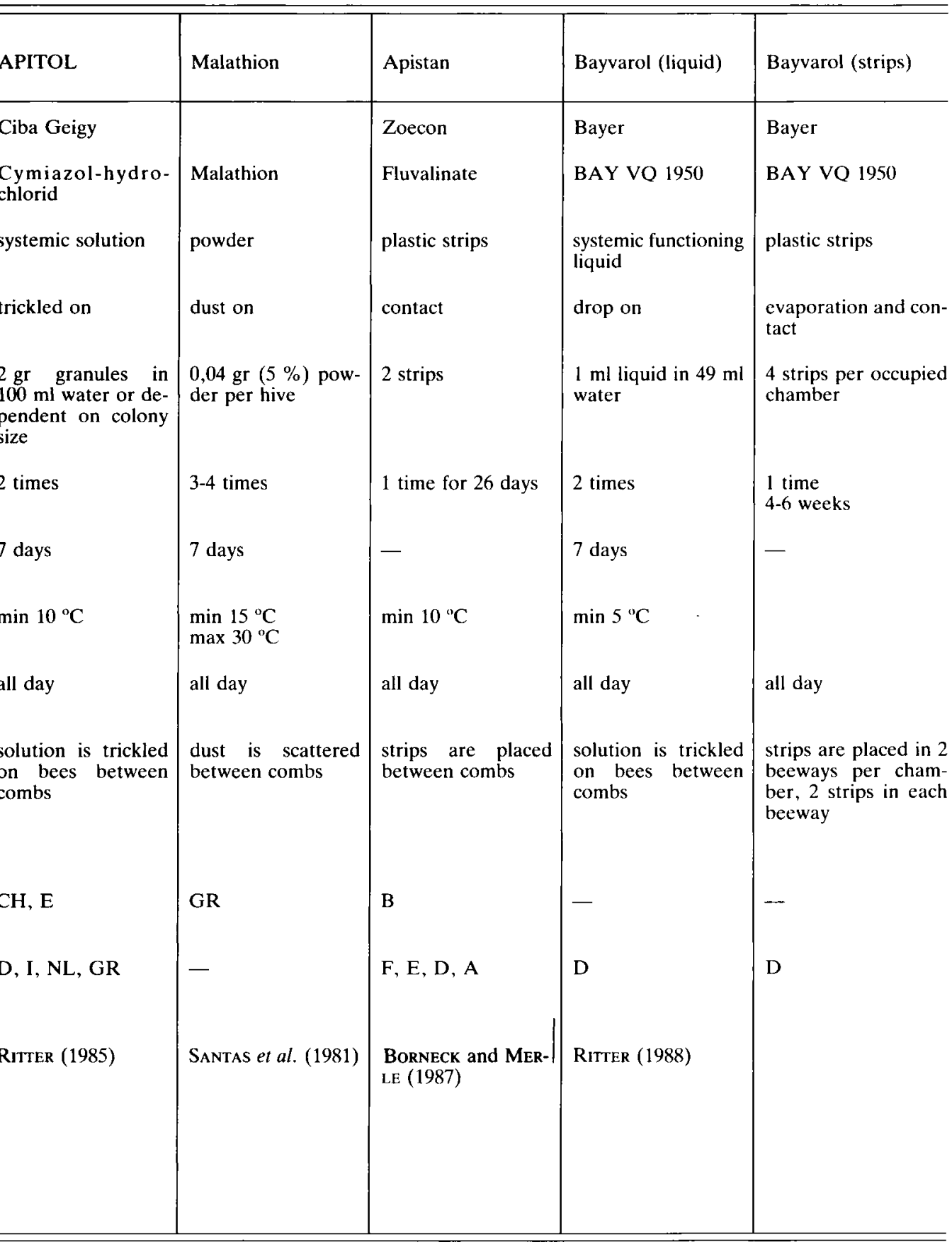


Parmi les nombreuses substances trouvées, seules quelques-unes ont débouché sur une utilisation dans la pratique apicole par les apiculteurs. Dans presque tous les pays d'Europe occidentale, les médicaments pour abeilles doivent répondre aux critères des médicaments vétérinaires. Leur éventuelle action toxique et mutagène pour l'homme est testée. En outre il faut rechercher les résidus éventuels dans les produits du rucher et tout danger potentiel pour l'utilisateur.

Le tableau présente les divers médicaments autorisés dans les différents pays ; une utilisation semblable a permis de les juger d'après les critères suivants : efficacité suffisante, bonne tolérance par les abeilles et absence de danger pour le consommateur et utilisateur.

Eingegangen im Juni 1988. Angenommen im Juni 1988 .

\section{REFERENCES}

Borneck R., Merle R., 1987. - Neue Bekämpfungsversuche der Varroatose mit Fluvalinat. Proc. XXXI Int. Congr. Apimondia, Warschau 1987, 105.

Colin M.E., Faucon J.P., Morand M., 1983. - Utilisation of aerosol to treat bee colonies against Varroatosis (Varroase). In : "Varroa jacobsoni Oud. affecting honeybees : present status and needs ", ed. R. Cavalloro, Rotterdam, 71-72.

RITrer W., 1985. - First trials with Apitol, a systemic functioning medicament. Proc. XXX Int. Congr. Apimondia, Nagoya 1985, Apimondia Publishing House, 198-200.

RITter W., 1986. - Versuche zur Entwicklung und Prüfung von Perizin, einem systemischen Medikament zur Bekämpfung der Varroatose der Honigbiene. Tierärztl. Umschau, 41 (2), 105-112.

Rutrer W., 1988. Control of Varroa jacobsoni with Bayvarol (liquid). (In preparation).

Rirter W., Perschil. F., 1983. - Prüfung der Wirkung von FOlbeX-VA-NEU (Isopropyl - 4, 4' dibrombenzilate) auf Varroamilben und der Verträglichkeit für Bienen. Apidologie, 14 (1), 9-27.

Santas L.A., Emmanouel N.G., Papadopoulou-Batzaki D.G., 1981. - Preliminary observations from the use of two control methods for Varroa disease in Greece. Proc. XXVIII Int. Congr. Apimondia, Acapulco 1981, Apimondia Publishing House, 356-360.

Wachendörfer G, Klepsch A., Stoya W., Kaiser E., 1985. - Derzeitiger Stand der medikamentellen Behandlung der Varroatose mit neuen Ameisensäureverdunstungssystemen. Allg. dtsch. Imkerztg., 19 (10), 300-306. 